\title{
Erratum
}

\section{Carbon-Carbon Double-Bond Isomerization and Diels-Alder Reaction of Dimethyl 5-Methylene-4-isopropylidene-2-cycloheptene-1,1-dicarboxylate with Dienophiles}

Ping Lu, Jinqiang Kuang, Shengming Ma* Synlett 2010, 227.

The advance online publicaton (e-First) version of this article contained wrong yields for compound 4e.

Those errors have been corrected for both the current online version and the print publication. 